\title{
Practical issues in medication compliance in hypertensive patients
}

\author{
This article was published in the following Dove Press journal: \\ Research Reports in Clinical Cardiology \\ 22 June 2011 \\ Number of times this article has been viewed
}

\author{
Jean-Marie Krzesinski ${ }^{1}$ \\ Marc Leeman ${ }^{2}$ \\ 'Nephrology Transplantation \\ Department, Domaine Universitaire, \\ Liège, Belgium; ${ }^{2}$ Department of \\ Internal Medicine and Hypertension \\ Clinic, Erasme University Hospital, \\ Brussels, Belgium
}

\begin{abstract}
Unsatisfactory compliance in the treatment of high blood pressure is frequently due to sequential barriers, such as insufficient patient education about the illness and low motivation to receive any treatment, existence of a large gap between physicians' perceptions of the problem and clinical reality, complexity of the treatment potentially generating adverse effects, and a health care environment with few public education campaigns and incentives for better coordinated supportive care. In order to improve drug compliance, establishment of personalized plans adapted to each patient is required. First, a good doctor-patient relationship is mandatory, with regular education of the patient about hypertension and its risks, discussion about adverse drug effects, and the complexity and cost of treatment. Second, to have any chance of success, the provider should offer convenient appointments and tailor the treatment regimen to the patient's lifestyle and needs, with written instructions. Third, there is a need to promote active patient collaboration with treatment. An innovative combination of home self-measurement of blood pressure, use of new technology options, eg, texting or telemedicine, and creation of a multidisciplinary working team can offer new, effective opportunities. This approach could reduce cardiovascular complications by improving the control of high blood pressure, and thereby the overall costs of hypertension to the health care system.
\end{abstract}

Keywords: arterial hypertension, therapeutic adherence, drug compliance, persistence

\section{Introduction}

High blood pressure is a major cardiovascular risk factor that affects approximately $25 \%$ of the adult population in industrialized countries. It is well known that effective control of hypertension markedly reduces the risk of cardiovascular events. ${ }^{1,2}$ In spite of this, in the US around $30 \%$ of patients treated with antihypertensive drugs do not achieve their target blood pressure. ${ }^{3}$ Poorly controlled hypertension confers a high risk of stroke, myocardial infarction, heart failure, and end-stage kidney disease. Nonadherence to antihypertensive therapy is believed to be a major cause of unsatisfactory blood pressure control. In a large cohort study of 10,447 patients with coronary artery disease, medication nonadherence was associated with uncontrolled blood pressure despite intensification of antihypertensive treatment, whereas high blood pressure became controlled over time in adherent patients. ${ }^{4}$

Medication-taking behaviors can be divided into two main concepts, namely adherence and persistence. ${ }^{5-7}$ Adherence is defined as appropriate use of therapy, including taking medications at the prescribed frequency, interval, and dosage. Adherence is reported as the percentage of prescribed doses taken per defined period of time, and may change over time. Persistence is defined as continuing the
Correspondence: Jean-Marie Krzesinski Nephrology Transplantation Department, University of Liège, CHU Sart

Tilman, Domaine Universitaire B35,

4000 Liège, Belgium

Tel +3243667203

Fax +32 43667205

Email jm.krzesinski@chu.ulg.ac.be 
use of medications for the specified treatment period and is measured in terms of time. Not surprisingly, these two medication-taking behaviors are related, ie, the lower the adherence, the higher the likelihood of early treatment discontinuation. ${ }^{8}$ The patient could also present with both patterns, ie, perfect adherence but nonpersistence with drug treatment or good persistence but bad execution (eg, drug holidays). Intentional and unintentional patterns of nonadherence have been recognized. ${ }^{7,9}$ Intentional nonadherence is an active process whereby the patient chooses to deviate from the treatment regimen. Unintentional nonadherence is a passive process whereby the patient may be careless or forgetful about adhering to the treatment regimen., ${ }^{7,9}$

\section{Measurement of medication adherence}

Methods for evaluation of adherence can be categorized as either direct or indirect. ${ }^{7,10}$ Neither of these methods is completely satisfactory. Direct methods include measurement of plasma drug concentrations. Plasma drug levels may be difficult to interpret, depending on the time interval between drug intake and when the blood sample is taken, drug pharmacokinetics, and interindividual differences in absorption and metabolism. Additionally, many patients typically increase their adherence in the few days before a scheduled visit to the clinic or laboratory. Finally, such tests are costly, so plasma drug levels are not usually measured in clinical practice.

Indirect methods are the most common approach used to measure treatment adherence. They include patient interview, questionnaire or diary, pill count, prescription refill, and electronic monitoring of medication use. Subjective evaluation by physicians tends to overestimate adherence and to detect poor adherence incorrectly. Responses to six-item or eight-item self-administered questionnaires have been shown to be reliable and closely associated with blood pressure control. ${ }^{11,12}$ Such self-reported measurement of medicationtaking can be a simple and inexpensive tool for detecting low adherence.

Counting pills allows calculation of the percentage of prescribed doses that have been taken between two visits. Pill counting can overestimate adherence because the patient may have discarded some tablets. In addition, the pattern of missed doses is not identified using this approach. Based on analysis of prescription refills derived from pharmacy data, the medication possession ratio and the proportion of days covered can be obtained, both methods relating the number of doses dispensed to the dispensing period. ${ }^{7}$ For example, if a 30-day supply dispensed on day 0 is used through day 40 , when the next 30-day supply is dispensed the medication possession ratio is $30 / 40$, ie, $75 \%$. In the literature, the medication possession ratio is often used as a dichotomous variable, with values $\geq 80 \%$ usually (and arbitrarily) considered as "good" adherence. ${ }^{13}$ However, the patient must use a pharmacy connected to the database. Electronic medication monitors are pill boxes equipped with a microprocessor which records the date and time of each opening of the container, hence providing accurate and detailed information on adherence. However, this method is expensive and generally restricted to clinical research. ${ }^{6}$

\section{Quantification of medication-taking behavior in hypertension}

Wide ranges of adherence rates in hypertension have been reported. The drug holiday phenomenon, ie, omission of medication on several sequential days, is frequent. Of more than 80,000 elderly Medicare beneficiaries ( $\geq 65$ years) with hypertension, $87 \%$ were adherent to at least one antihypertensive drug and $72 \%$ were adherent to their full regimen. ${ }^{14}$ Adherence was defined as proportion of days covered $\geq 80 \%$ and measured using automated pharmacy data. ${ }^{14}$ An Italian study including 18,806 newly diagnosed hypertensive patients showed that, although the adherence level varied with time, $8 \%, 41 \%$, and $51 \%$ of the patients were classified as high (adherence $>80 \%$ ), intermediate (adherence 40\%-79\%), and low (adherence $<40 \%$ ) adherers, respectively, at the beginning of the study. ${ }^{15}$ Such variable rates may be due to the various methods used to assess adherence, to differences in the populations studied, to the antihypertensive agents used, and because adherence changes over time. Persistence is also reported variably. Typically, about $50 \%$ of patients discontinue their antihypertensive therapy after 1 year. ${ }^{5,8}$ At 10 years, a persistence rate of $39 \%$ has been reported. ${ }^{16}$

\section{Factors related to medication- taking behavior in hypertension}

Adherence is a dynamic process. It is higher in the days following a visit to the physician and in the days preceding the next visit, displaying a U-shaped curve referred to as "white coat adherence". Numerous barriers contribute to lack of adherence/persistence with antihypertensive medication (see Table 1). ${ }^{5,6,10}$ Insufficient interaction between the patient and the physician may result in poor awareness on the part of the patient of the complications of hypertension and the benefits of its treatment. Adherence and persistence are typically low in the treatment of asymptomatic chronic diseases with drugs 
Table I Factors contributing to low adherence/persistence with antihypertensive drugs

\begin{tabular}{ll}
\hline Category & Examples \\
\hline $\begin{array}{l}\text { Patient-physician } \\
\text { relationship }\end{array}$ & $\begin{array}{l}\text { Poor communication } \\
\text { Insufficient patient information/education } \\
\text { Physical/cognitive impairments (vision problems, } \\
\text { dementia) } \\
\text { Asymptomatic } \\
\text { Condition }\end{array}$ \\
Lifelong treatment \\
No immediate consequences of stopping therapy \\
Adverse effects \\
Complexity of regimen \\
Cost of medication \\
Unemployment/poverty \\
Lack of insurance \\
Lack of transportation \\
Social deprivation \\
Inadequate health care coverage \\
Difficult access to health care \\
Lack of continuity of care
\end{tabular}

that are costly and potentially have adverse effects. Adherence decreases proportionately to frequency of dose and therapeutic complexity. ${ }^{10,17}$ Several reports have shown that adherence and persistence rates are higher with renin-angiotensin blockers and lower with diuretics. ${ }^{5,6} \mathrm{~A}$ large study evaluated persistence in 242,882 newly treated patients receiving monotherapy. After 1 year, the proportion of patients still taking the initially prescribed medication was $52 \%$ for angiotensin II receptor blockers, $48 \%$ for angiotensin-converting enzyme inhibitors, $40 \%$ for beta-blockers, $38 \%$ for calcium antagonists, and 30\% for diuretics. ${ }^{18}$ Compared with patients receiving diuretics, those treated with other antihypertensive agents were significantly less likely to discontinue therapy. ${ }^{18} \mathrm{~A}$ recent metaanalysis confirms highest adherence to angiotensin II receptor blockers and angiotensin-converting enzyme inhibitors, and lowest adherence to diuretics and beta-blockers. ${ }^{19}$ In a study of 588 hypertensive patients, intentional nonadherence was reported by $9 \%$ of the participants, compared with $31 \%$ who reported unintentional nonadherence. ${ }^{9}$ Intentional nonadherence was more likely to be reported by nonwhite patients, patients without diabetes, and patients who reported at least five adverse effects they attributed to their antihypertensive medication. ${ }^{9}$ Socioeconomic factors, such as lack of insurance coverage, have also been related to adherence and blood pressure control. ${ }^{20,21}$

\section{Consequences of poor adherence}

Numerous studies indicate that low adherence to antihypertensive drugs affects blood pressure control, rate of complications, and overall health care resource utilization and costs. ${ }^{4-6,10,14}$ Few large studies have related adherence to antihypertensive medication with hard endpoints. In the study of 18,806 newly diagnosed hypertensive patients discussed earlier, high adherers (adherence $>80 \%$ ) had a significantly lower risk of cardiovascular events in comparison with low adherers (adherence $<40 \%$ ) after an average follow-up duration of 4.6 years..$^{15}$ A Canadian study reported a $22 \%$ reduction in stroke incidence in patients with high adherence (medication possession ratio $\geq 80 \%$ ) compared with patients with lower adherence in a cohort of 83,267 newly treated hypertensive patients. ${ }^{22}$ More recently, an Italian populationbased prospective cohort study of 242,594 patients showed a significant increase in the incidence in coronary and cerebrovascular events in patients who discontinued treatment and in patients with low drug coverage, after a mean follow-up of 6 years. ${ }^{23}$

The impact of adherence on blood pressure control has been challenged. ${ }^{24}$ Some concern has been raised that the association between low medication adherence and adverse outcomes may be, at least in part, related to a "healthy adherer" effect. The healthy adherer effect implies that the lower risk of adverse outcomes associated with adherence may be a surrogate marker for overall healthy behavior. ${ }^{25}$ For example, data from the Candesartan in Heart Failure Assessment of Reduction in Mortality and Morbidity (CHARM) study in patients with congestive heart failure showed that patients with a high rate of adherence to placebo had better outcomes, including mortality, compared with patients with low adherence to the active treatment. ${ }^{26}$ It is hypothesized that patients who take their medication regularly are also more likely to perform other healthy behaviors, such as eating properly and exercising regularly. ${ }^{7}$ Hence, there is widespread agreement to promote healthy lifestyle habits as well as to overcome barriers to good medication-taking behavior in the hope of reducing the burden of cardiovascular disease. ${ }^{1}$

\section{Improving adherence to antihypertensive medication}

As mentioned earlier, poor medication adherence is an enormous public health problem. Whatever the patient's age, gender, race, or comorbidities, lowering blood pressure will be beneficial at every stage of the cardiovascular continuum, and the target should be $<140 / 90 \mathrm{mmHg}$ in all adult patients younger than 80 years. Initiating drug treatment is useless if the tablets are unused, and identification of this as a potential problem is crucial. A combinational approach is often needed to increase the compliance rate. 
There are three important steps to improve drug adherence. We have to prevent noncompliance, to detect it, and to improve and/or maintain vigilance. However, there are sequential barriers to drug adherence in hypertension, ie, poor communication, low motivation, complexity of treatment, acute side effects (influence on adherence) but also delayed side effects, limited long-term motivation, and reinforcement of poor habits (influence on persistence).

In the first place, to detect noncompliance, there is a need to talk about its possible existence (increase awareness of the problem) and to monitor treatment whenever possible. Several recommendations can be made to improve this problem when identified. There are at least four important determining influences on compliance which should be recognized and brought under control:

- The patient (hypertension is seen as an unavoidable consequence of old age or of anxiety, an asymptomatic disorder, and therefore perceived as benign)

- The health provider (not aware of and not trained to test for the problem)

- The treatment (drug price, undesirable adverse effects)

- The therapeutic environment (complexity of the regimen).

Gallup and Cotugno ${ }^{27}$ showed that treatment for high blood pressure was stopped by patients because of side effects in $11 \%$ of cases, thinking that the doctors asked them to do this in $25 \%$, believing themselves to be cured (because they were without symptoms) in $46 \%$, and for financial reasons in $6 \%$.

Consistent control of blood pressure requires that patients with high blood pressure follow dietary regimens and drug treatment. Dietary changes, especially reduction of salt and excess weight, are crucial in the management of the hypertensive population. This approach can decrease the need for antihypertensive treatment. This is important because antihypertensive therapy may have potential side effects, whereas high blood pressure is very often asymptomatic. Therefore, limiting the number of agents needed to control blood pressure by dietary and lifestyle measures is an interesting approach. However, lifestyle advice is generally not followed in the long term, except if the patient is motivated. Compliance with dietary therapy is better, and success rates in achieving blood pressure control are higher when accompanied by active patient guidance or counseling by clinicians and ancillary medical personnel with expertise in dietary management. Less than one-third of treated patients change their dietary habits or increase their physical activity, and in this situation, persistence with the drug treatment is likely to be suboptimal.
To achieve better compliance, we need to improve the relationship between the patient and the health care provider and to integrate this into the health care system. Patients need to know the risks associated with their hypertension and to consider them as serious, to believe in the efficacy of their treatment, to be motivated by well defined goals, and to know the effects expected from therapy.

In addition to improvement of the health care system with guidelines to increase adherence, the creation of a medical treatment team, supporting public education campaigns and incentives for better coordinated support care, patient/ provider education, and engagement should be better developed. An approach to improve compliance must draw on aspects of educational, behavioral, affective, and provider intervention strategies.

\section{The patient}

Educating and involving patients is crucial to motivating them and making them aware of the necessity for treatment. Each patient is an individual, and the goal of patient-centered approaches to improve compliance with antihypertensive therapy is to identify each patient's barriers to compliance and develop personalized self-management plans. Adherence could be enhanced further still when the patient is empowered to become involved in medical decisions and monitoring their care. There is a diverse range of patient-related factors that can affect compliance with antihypertensive therapy. The importance of any particular factor will differ between individuals and also in the same individual over time. The asymptomatic nature of hypertension is particularly important, because this is likely to influence the perceived importance of other patient-related factors.

An important point cited by the European Society of Hypertension guidelines in 2007 is the crucial role of patient information on the risk of hypertension and the benefits of treating it. Patient understanding of the long-term health implications and the asymptomatic nature of uncontrolled hypertension is likely to improve compliance with antihypertensive therapy. Patient education can be achieved in many ways, including verbal, written, and visual approaches, or a combination of these approaches. Whichever approach is utilized, the language used should be clear and free of jargon. Instructions should be simple and unambiguous.

\section{The health care provider}

The role of the health care provider is particularly important in the field of drug compliance. First, the health care provider 
must propose tight follow-up with frequent office visits. Office visits provide an important opportunity to reinforce hypertension-related educational messages. Clinicians could do more to underscore the importance of medication adherence and healthy living to their hypertensive patients. Moreover, the health care provider must be trained in the problem of nonadherence.

Good communication and interpersonal skills and regular appointments are examples of health care provider interventions that can foster a collaborative relationship with patients and improve patient compliance with antihypertensive therapy. Provider interventions for improving compliance are ultimately intended to achieve a collaborative and interactive partnership between health care provider and patient, whereby they work together to control blood pressure. However, there is a large gap between physicians' perceptions and clinical reality when it comes to assessing the success of therapy, which is often overestimated. Physicians are of course fully aware that compliance in hypertension often tails off substantially within the first year of treatment.

The health care provider must be very attentive to identify problems which could interfere with adherence. Some depressive conditions could exist, leading to, for example, missed medical appointments. It is thus important to identify and contact patients who are not showing up to consultations and to focus on patients in whom therapeutic goals are not reached. Convenient appointments need to be given, the treatment regimen should be tailored to the patient's lifestyle and needs, simplified or adapted if necessary, individualized written instructions should be provided, and the patient given encouragement to collaborate in their treatment (see Table 2).

Table 2 Ten guidelines for the physician to improve antihypertensive drug compliance

I. Educate the patient about hypertension and its treatment with clear and accepted goals

2. Be easily available to communicate regularly with the patient

3. Keep the treatment as simple and cheap as possible with written information

4. Prescribe considering the patient and potential drug interactions

5. Avoid adverse drug effects by starting the treatment at a low dose

6. Stop any inefficient or badly tolerated treatment and avoid therapeutic inertia

7. Combine efficient and well tolerated drugs in the same pill

8. Stimulate the patient to be active in medical management

9. Create a multidisciplinary team to improve family and community support

10. Use all possible modern reminder aids to ensure daily drug intake

\section{The treatment}

Simplification of the treatment regimen is important. A oncedaily dosing regimen is useful. Eisen et $\mathrm{al}^{28}$ showed that compliance decreased from $84 \%$ for a single daily dose to $59 \%$ for a three times daily dosage. Because monotherapy is usually insufficient to control blood pressure, physicians are increasingly using two or more antihypertensive agents concurrently. When this is the case, fixed-dose combinations deliver agents from different therapeutic classes to provide effective blood pressure reduction. The medications are packaged in a convenient format for use in a simple treatment regimen. This can help to overcome the adherence issue. ${ }^{29}$ Minimizing the total number of daily doses has been found to be more important in promoting adherence, and perhaps more importantly persistence with treatment, than minimizing the total number of medications. ${ }^{30}$ Yet this approach seems not always sufficient. For instance, a longitudinal database study of 4783 patients prescribed once-daily antihypertensive treatment in 1989-2006 reported that half had stopped taking their prescribed treatments within 1 year. $^{8}$

Side effects must also be monitored. Great attention must be paid at each medical contact to acknowledge drug side effects (even subtle) and one must be prepared to change drug doses or types of drug if needed. Tolerability is one of the main factors determining adherence, because it has a major impact on the probability of treatment discontinuation. The angiotensin receptor blockers (and probably also the new renin inhibitors) are the safest and best tolerated class of antihypertensive drugs, and therefore their use increases the chance that a patient will persist with antihypertensive treatment over time. In a retrospective cohort study of a large patient population $(\mathrm{n}=21,723)$, Bloom $^{31}$ showed that angiotensin receptor blockers had the highest rate of persistence (64\%) at 1 year. This was confirmed at 4 years (51\%) by a longitudinal analysis of a subset of this study cohort $(n=15,175)$. Persistence with angiotensin receptor blockers was significantly greater than when using other classes of antihypertensives. $^{32}$

Another way to improve blood pressure control, without dealing directly with compliance, is to use more "forgiving" antihypertensive agents with longer half-lives, which will probably help to maintain a more stable blood pressure, despite imperfect adherence. The physician should choose the agent with the longest half-life in each class. These forgiving drugs should have efficacy which is unaffected by delayed or missed doses. However, thiazide diuretics, although less widely used due to potential side effects, are among the most powerful in this respect. Moreover, such treatment 
is cheap. Thiazide diuretics should also be considered when prescribing a drug. In chronic disease, when drug treatment is proposed for a long period of time, it is very important to consider compliance in the evaluation of the cost of medication. Expensive medications are one of the treatment characteristics which reduce adherence, and probably persistence with therapy even more.

Another approach in antihypertensive prescribing could be initiation of low-dose combination therapy, as proposed by a Canadian group. ${ }^{33}$ They suggest starting initial antihypertensive therapy with a low-dose angiotensinconverting enzyme inhibitor-diuretic combination or an angiotensin receptor blocker-diuretic combination. The combination therapy can then be uptitrated to the highest dose, if necessary. This simplified antihypertensive algorithm using such initial low-dose, fixed-dose combination therapy could be superior to guideline-based practice for the management of hypertension.

\section{The therapeutic environment}

Education of the patient and the health care provider will never be enough to solve the problem of nonadherence. ${ }^{34}$ Patients have many reasons for missing their treatment but they do not always share these with their physician. Discussing these reasons takes time, but someone must explore the patient's beliefs about antihypertensive medication and persuade them that the benefits outweigh any worries they may have. One reason could be that, in most health care systems, physicians are not given enough incentives or resources to control blood pressure effectively. This is indeed a timeintensive intervention. A recent paper ${ }^{35}$ indicates that "pay for performance" has no effect on hypertension-related clinical outcomes. Quality of care in the UK was not modified by introducing such financial measures. However, our task as health care providers is to promote awareness of the dangers of hypertension across government, the public, health professionals, carers, and perhaps even schoolchildren, who will be the next generation of hypertensive people if no action is taken. Because hypertension is a chronic disease, it is also important to maintain and even improve drug adherence by supervising treatment and positively reinforcing compliance, perhaps involving a family member. The goal of behavioral approaches is to modify compliance by targeting, shaping, and reinforcing behavior patterns that improve compliance with antihypertensive therapy. Behavioral approaches can be implemented by means of simplifying treatment schedules, using reminder techniques, modifying drug packaging, and encouraging self-monitoring of blood pressure by patients.
Affective intervention approaches are useful to improve compliance with antihypertensive therapy. These approaches focus on feelings and emotions, and often rely on family relationships and social networks. Affective interventions can be implemented by means of social support networks, involvement and education of family members, counseling, home visits, and nurse telephone calls. Creation of efficient medical teams should be encouraged. Patients may respond more positively to various aspects of a message depending on the messenger. Nurses, for instance, can spend more time with patients and reiterate the message in alternative ways. They can also reinforce drug compliance by using the short message service. Text messages could hold potential for improving adherence to some drug regimens. This kind of intervention has been shown to be useful in improving drug compliance in patients suffering from chronic infectious diseases, such as tuberculosis. ${ }^{36}$ The pharmacist can undertake regular medication reviews, do blood pressure monitoring, educate the patient on avoidance of some side effects and on improving drug efficacy, by giving advice on how and when the drug should be taken, and could alert the physician when repeat prescriptions appear ineffective or are not collected by the patient. This "multidisciplinary team" could be very effective in improving blood pressure control. ${ }^{37}$ Patients in whom hypertension is managed by a physician-pharmacist team have lower blood pressure levels, and are more likely to reach their target blood pressure than patients treated by a physician alone. ${ }^{38}$

Patients should be active participants in their own treatment. This needs therapeutic education enabling hypertensive patients to manage their illness with the help of health partners. For example, patients should be encouraged to monitor their own blood pressure regularly at home. Edmonds et $\mathrm{al}^{39}$ monitored 37 patients with high blood pressure treated for 3 months. They had been taught self-measurement, and their compliance was $65 \%$ at the beginning of the trial and $81 \%$ after 3 months. Of those who were noncompliant prior to the study, adherence was achieved in $70 \%$ of cases after self-measurement. This approach could also motivate patients to adhere to both lifestyle changes and medication. Of eleven randomized controlled trials, six (54\%) reported a significant improvement in compliance attributable to self-measurement of blood pressure. ${ }^{40}$ In this way, control of blood pressure was higher, ${ }^{41}$ and the patients were more satisfied by this approach, which did not increase the cost of treatment. ${ }^{42}$ The success of such an approach is even greater if self-monitoring, Internet support, and pharmacy management are used together. ${ }^{43}$ Therefore, an innovative combination of self-management, 
using an alarm clock function on a mobile phone and new technology eg, short reminder message services and telemedicine, as well as a multidisciplinary approach offer new therapeutic possibilities. It has also been shown recently that home blood pressure telemonitoring could be helpful in addressing the problem of compliance. ${ }^{44}$

\section{Conclusion}

No single intervention strategy is superior for improving drug compliance, and complex methods are needed to identify individual barriers to compliance and help to develop personalized self-management plans. To prevent, detect, and improve the problem of drug adherence, we must give convenient appointments, simplify and adapt treatment with individualized instructions, and promote patient collaboration with treatment. We must also discuss compliance, monitor treatment whenever possible, identify and contact patients who are not attending their appointments, and focus on patients in whom therapeutic goals are not being reached. Our role as doctors is to supervise treatment, provide feedback on treatment to the patient, and involve family members, nurses, and pharmacists to reinforce compliance.

\section{Disclosure}

The authors report no conflicts of interest in this work.

\section{References}

1. Mancia G, De Backer G, Dominiczak A, et al. 2007 guidelines for the management of arterial hypertension: The task force for the management of arterial hypertension of the European Society of Hypertension and of the European Society of Cardiology. J Hypertens. 2007;25: $1105-1187$.

2. Chobanian AV, Bakris GL, Black HR, et al. The seventh report of the Joint National Committee on prevention, detection, evaluation, and treatment of high blood pressure. JAMA. 2003;289:2560-2572.

3. Egan BM, Zhao Y, Axon RN. US trends in prevalence, awareness, treatment and control of hypertension, 1998-2008. JAMA. 2010;303: 2043-2050.

4. Ho PM, Magid DJ, Shetterly SM, et al. Importance of therapy intensification and medication nonadherence for blood pressure control in patients with coronary disease. Arch Intern Med. 2008;168:271-276.

5. Elliott WL. Improving outcomes in hypertensive patients: Focus on adherence and persistence with antihypertensive therapy. $J$ Clin Hypertens. 2009;11:376-382.

6. Burnier M. Medication adherence and persistence as the cornerstone of effective antihypertensive therapy. Am J Hypertens. 2006;19: 1190-1196.

7. Ho PM, Bryson CL, Rumsfeld JS. Medication adherence: Its importance in cardiovascular outcomes. Circulation. 2009;119:3028-3035.

8. Vrijens B, Vincze G, Kristanto P, Urquhart J, Burnier M. Adherence to prescribed antihypertensive drug treatments: Longitudinal study of electronically compiled dosing histories. BMJ. 2008;336:1114-1117.

9. Lowry KP, Dudley TK, Oddone EZ, Bosworth HB. Intentional and unintentional nonadherence to antihypertensive medication. Ann Pharmacother. 2005;39:1198-1203.

10. Osterberg L, Blaschke T. Adherence to medication. New Engl J Med. 2005;353:487-497.
11. Mulazzi I, Cambou JP, Girerd X, Nicodeme R, Chamontin B, Amar J. Six-item self-administered questionnaires in the waiting room: An aid to explain uncontrolled hypertension in high-risk patients seen in general practice. J Am Soc Hypertens. 2009;3:221-227.

12. Morisky DE, Ang A, Krousel-Wood M, Ward HJ. Predictive value of a medication adherence measure in an outpatient setting. $J$ Clin Hypertens. 2008;10:348-354.

13. Halpern MT, Khan ZM, Schmier JK, et al. Recommendations for evaluating compliance and persistence with hypertension therapy using retrospective data. Hypertension. 2006;47:1039-1048.

14. Fung V, Huang J, Brand R, Newhouse JP, Hsu J. Hypertension treatment in a Medicare population: Adherence and systolic blood pressure control. Clin Ther. 2007;29:972-984.

15. Mazzaglia G, Ambrosioni E, Alacqua M, et al. Adherence to antihypertensive medications and cardiovascular morbidity among newly diagnosed hypertensive patients. Circulation. 2009;120:1598-1605.

16. Van Wijk BL, Klungel OH, Heerdink ER, de Boer A. Rate and determinants of 10-year persistence with antihypertensive drugs. J Hypertens. 2005;23:2101-2107.

17. Choudhry NK, Fischer MA, Avorn J, et al. The implications of therapeutic complexity on adherence to cardiovascular medications. Arch Intern Med. 2011;171: 814-822.

18. Patel BV, Remigio-Baker RA, Mehta D, Thiebaud P, Frech-Tamas F, Preblick R. Effects of initial antihypertensive drug class on patient persistence and compliance in a usual-care setting in the United States. J Clin Hypertens. 2007;9:692-700.

19. Kronish IM, Woodward M, Sergie Z, Ogedegbe G, Falzon L, Mann DM. Meta-analysis: Impact of drug class on adherence to antihypertensives. Circulation. 2011;123:1611-1621.

20. Duru OK, Vargas RB, Kermah D, Pan D, Norris KC. Health insurance status and hypertension monitoring and control in the United States. Am J Hypertens. 2007;20:348-353.

21. Bautista LE. Predictors of persistence with antihypertensive therapy: Results from the NHANES. Am J Hypertens. 2008;21: 182-188.

22. Kettani FZ, Dragomir A, Côté R, et al. Impact of better adherence to antihypertensive agents on cerebrovascular disease for primary prevention. Stroke. 2009;40:213-220.

23. Corrao G, Parodi A, Nicotra F, et al. Better compliance to antihypertensive medications reduces cardiovascular risk. J Hypertens. 2011;29: 610-618.

24. Wetzels GEC, Nelemans P, Schouten JS, Prins MH. Facts and fiction of poor compliance as a cause of inadequate blood pressure control: A systematic review. J Hypertens. 2004;22:1849-1855.

25. Simpson SH, Eurich DT, Majumdar SR, et al. A meta-analysis of the association between adherence to drug therapy and mortality. $B M J$. 2006;333:15-20.

26. Granger BB, Swedberg K, Ekman I, et al. Adherence to candesartan and placebo and outcomes in chronic heart failure in the CHARM programme: Double-blind, randomised, controlled trial. Lancet. 2005;366:2005-2011.

27. Gallup G Jr, Cotugno HE. Preferences and practices of Americans and their physicians in antihypertensive therapy. Am J Med. 1986; 81 Suppl 6C:20-24.

28. Eisen SA, Woodward RS, Miller D, Spitznagel E, Windham CA. The effect of medication compliance on the control of hypertension. J Gen Intern Med. 1987;2:298-305.

29. Schroeder K, Fahey T, Ebrahim S. How can we improve adherence to blood pressure-lowering medication in ambulatory care? Systematic review of randomized controlled trials. Arch Intern Med. 2004;164: 722-732.

30. Iskedijan M, Einarson TR, MacKeigan LD, et al. Relationship between daily dose frequency and adherence to antihypertensive pharmacotherapy: Evidence from a meta-analysis. Clin Ther. 2002;24: 302-316.

31. Bloom BS. Continuation of initial antihypertensive medication after 1 year of therapy. Clin Ther. 1998;20:671-681. 
32. Conlin PR, Gerth WC, Fox J, Roehm JB, Boccuzzi SJ. Four-year persistence patterns among patients initiating therapy with the angiotensin II receptor antagonist losartan versus other antihypertensive drug classes. Clin Ther. 2001;23:1999-2010.

33. Feldman RD, Zou GY, Vandervoort MK, Wong CJ, Nelson SAE, Feagan BG. A simplified approach to the treatment of uncomplicated hypertension. A cluster randomized, controlled trial. Hypertension. 2009;53:646-653.

34. Home R, Clatworthy J, Polmear A, Weinman J. Do hypertensive patients' beliefs about their illness and treatment influence medication adherence and quality of life? J Hum Hypertens. 2001;15 Suppl 1: S65-S68.

35. Serumaga B, Ross-Degan D, Avery AJ, et al. Effect of pay for performance on the management and outcomes of hypertension in the United Kingdom: Interrupted time series study. BMJ. 2011;342:108.

36. Barclay E. Text messages could hasten tuberculosis drug compliance. Lancet. 2009;373:15-16.

37. Walsh JM, McDonald KM, Shojania KG, et al. Quality improvement strategies for hypertension management: A systematic review. Med Care. 2006;44:646-657.

38. Carter BL, Ardery G, Dawson JD, et al. Physician and pharmacist collaboration to improve blood pressure control. Arch Intern Med. 2009;169:1996-2002.
39. Edmonds D, Foerster E, Groth H, Gremminger P, Siegenthaler W, Vetter W. Does self-measurement of blood pressure improve patient compliance in hypertension. J Hypertens.1985;3(Suppl 1): S31-S34.

40. Ogedegbe $\mathrm{G}$, Schoenthaler A. A systemic review of the effects of home blood pressure monitoring on medication adherence J Clin Hypertens. 2006;8:174-180.

41. Marques-Contreras E, Martell-Claros N, Gil-Guillen V, et al. Efficacy of a home blood pressure monitoring programme on therapeutic compliance in hypertension: The EAPACUM-HTA study. J Hypertens. 2006;24:169-175.

42. McManus RJ, Mant J, Roalfe A, et al. Targets and self monitoring in hypertension: Randomised controlled trial and cost effectiveness analysis. BMJ. 2005;331:493

43. Jones DW, Peterson Ed. Improving hypertension control rates: Technology, people, or systems? JAMA. 2008;299:2896-2898.

44. Parati G, Omboni S. Role of home pressure telemonitoring in hypertension management: An update. Blood Press Monit. 2010;15(6): 285-295.

\section{Publish your work in this journal}

Research Reports in Clinical Cardiology is an international, peerreviewed, open access journal publishing original research, reports, editorials, reviews and commentaries on all areas of cardiology in the clinic and laboratory. The manuscript management system is completely online and includes a very quick and fair peer-review system.
Visit http://www.dovepress.com/testimonials.php to read real quotes from published authors. 